\title{
Consideraciones Anatómicas en la Parotidectomía: Revisión de la Literatura a Propósito de un Caso
}

\author{
Anatomical Considerations in Parotidectomy: Review of the Literature and Case Report
}

\author{
Ignacio Velasco*; Felipe Salinas**; Leonardo Aguilar ${ }^{* * *}$; Alejandra Gallego****; Juan Pastrián ${ }^{* * * * *}$; \\ Rodrigo Fariña $^{* * * * *}$; Reinaldo Soto ${ }^{* * *}$; Cristina Mebus ${ }^{* * * * * *}$ \& Alejandro Zurbuchen ${ }^{* * * * * *}$
}

\begin{abstract}
Velasco, I.; SAlinas, F.; AgUilar, L.; GAllego, A.; PASTrián, J.; FARIÑA, R.; SOTO, R.; MEBUS, C. \& ZURBUCHEN, A. Consideraciones anatómicas en la parotidectomía: revisión de la literatura a propósito de un caso. Int. J. Morphol., 31(1):231-238, 2013.

RESUMEN: La parotidectomía es un procedimiento quirúrgico habitualmente realizado por Cirujanos Maxilofaciales y Cirujanos de Cabeza y Cuello, en el cual se remueve parcialmente o en su totalidad a la glándula parótida. Las indicaciones más comunes para éste procedimiento son las neoplasias de la glándula. Aproximadamente el $80 \%$ del total de los tumores de glándulas salivales ocurren en la parótida. De estos, el 75-80\% son de naturaleza benigna. Sin embargo, la parotidectomía es un procedimiento sumamente complejo debido a la anatomía regional y por la íntima relación de la glándula parótida con el nervio facial. Siendo la clave de este procedimiento la preservación funcional de éste nervio y de sus ramos. Por lo tanto el conocimiento acabado de la anatomía de la glándula parótida y de sus estructuras anexas es necesario para el éxito quirúrgico. Esta revisión y reporte de caso discutirá las consideraciones anatómicas que deben tenerse durante la parotidectomía para disminuir la posibilidad de alguna complicación.
\end{abstract}

PALABRAS CLAVE: Glándula parótida; Región parotídea; Parotidectomía; Nervio facial; Tumor de Warthin.

\section{INTRODUCCIÓN}

Los cánceres de glándulas salivales son aproximadamente el $3 \%$ de todas las lesiones malignas de cabeza y cuello diagnosticadas en los Estados Unidos cada año; la mayoría de éstas se localizan en la glándula parótida (GP). Aproximadamente el $80 \%$ de los tumores de glándulas salivales ocurren en la GP. De éstos, el 75-80\% son de naturaleza benigna y de origen epitelial (Futran et al., 2009).

La parotidectomía es un procedimiento quirúrgico habitualmente realizado por Cirujanos Maxilofaciales y Cirujanos de Cabeza y Cuello, en el cual se remueve parcialmente o en su totalidad a la GP. Las indicaciones más comunes para este procedimiento son las neoplasias de la GP o las metástasis a los linfonodos parotídeos (Leverstein et al., 1997).

Sin embargo, la parotidectomía es un procedimiento sumamente complejo debido a la anatomía regional y a la íntima relación que posee la GP con el nervio facial (NF). Siendo la clave de este procedimiento la preservación funcional de éste nervio, a través de una correcta identificación durante la cirugía (Pather \& Osman, 1996).

Por lo tanto, el conocimiento acabado de la anatomía de la GP y de sus estructuras anexas es necesario para el éxito de la parotidectomía. El objetivo de esta revisión y reporte de caso es discutir las consideraciones anatómicas de la parotidectomía para disminuir la posibilidad de alguna complicación relacionada a la cirugía.

Consideraciones anatómicas en la parotidectomía. Las glándulas parótidas son el par de glándulas salivales más grandes y están ubicadas en la región preauricular (Fig. 1), limita por superior con el arco cigomático, por anterior puede prolongarse sobre el músculo masetero junto con su conducto excretor, el conducto parotídeo (Stenon) que atrave-

\footnotetext{
Residente. Departamento de Cirugía Oral y Maxilofacial, Universidad de Puerto Rico, Puerto Rico, E.E.U.U.

** Residente. Programa de Cirugía Bucal y Maxilofacial, Universidad de Los Andes, Santiago, Chile.

*** Profesor, Departamento de Cirugía Bucal y Maxilofacial, Universidad de Los Andes, Santiago, Chile.

**** Servicio de Cirugía de Cabeza y Cuello, Hospital del Salvador, Santiago, Chile.

*****Servicio de Cirugía Maxilofacial, Hospita del Salvador, Santiago, Chile.
} 


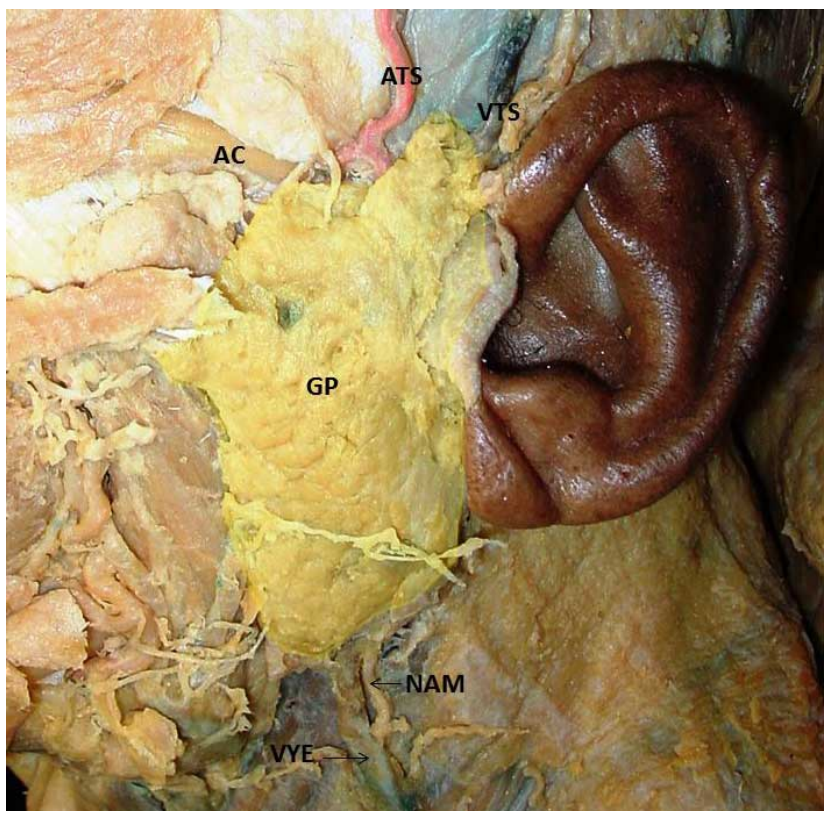

Fig. 1. Disección facial y cervical donde se observa glándula parótida (GP), arco cigomático (AC), arteria temporal superficial (ATS), vena temporal superficial (VTS), nervio auricular mayor (NAM) y vena yugular externa (VYE).

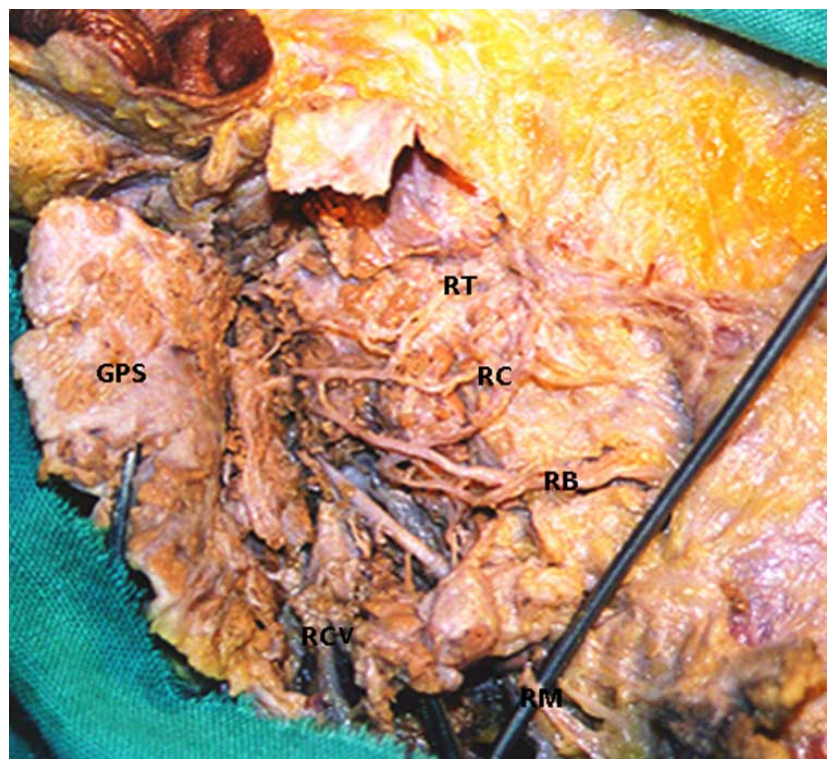

Fig. 2. Disección de GP, se retrae lobo superficial de GP (GPS) para observar NF y sus ramos: ramo frontotemporal (RT), ramo cigomático (RC), ramo bucal (RB), ramo marginal mandibular (RM) y ramo cervical (RCV).

sará el músculo buccinador a nivel del segundo molar maxilar para llegar a la cavidad oral. Medialmente la glándula se comunica con el espacio perifaríngeo. El polo inferior de la GP se extiende hasta el margen anteromedial del músculo esternocleidomastoideo (ECM). La GP anatómicamente se encuentra dividida en 2 lobos: superficial y profundo, debido a que es atravesada por el NF (Fig. 2).
La parotidectomía es un procedimiento que se realiza bajo anestesia general. Se tratan de evitar agentes paralíticos de larga duración para permitir la monitorización del NF. Éste nervio craneal (VII par) es sumamente complejo ya que puede presentar múltiples variaciones, por lo que el conocimiento de sus principales ramas y de su correspondiente inervación a los músculos faciales es de suma importancia para el manejo quirúrgico de la GP.

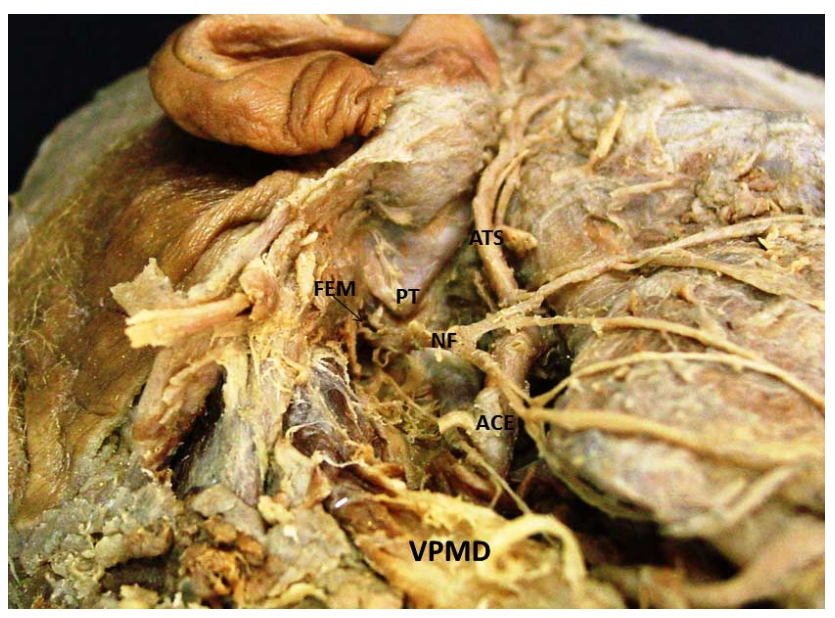

Fig. 3. Disección facial, visión inferior. Se extrajo GP para para observar nervio facial (NF) saliendo del foramen estilomastoideo (FEM) y de sus relaciones con el puntero tragal (PT) y el vientre posterior del musculo digástrico (VPMD). Arteria carótida externa (ACE), arteria temporal superficial (ATS).

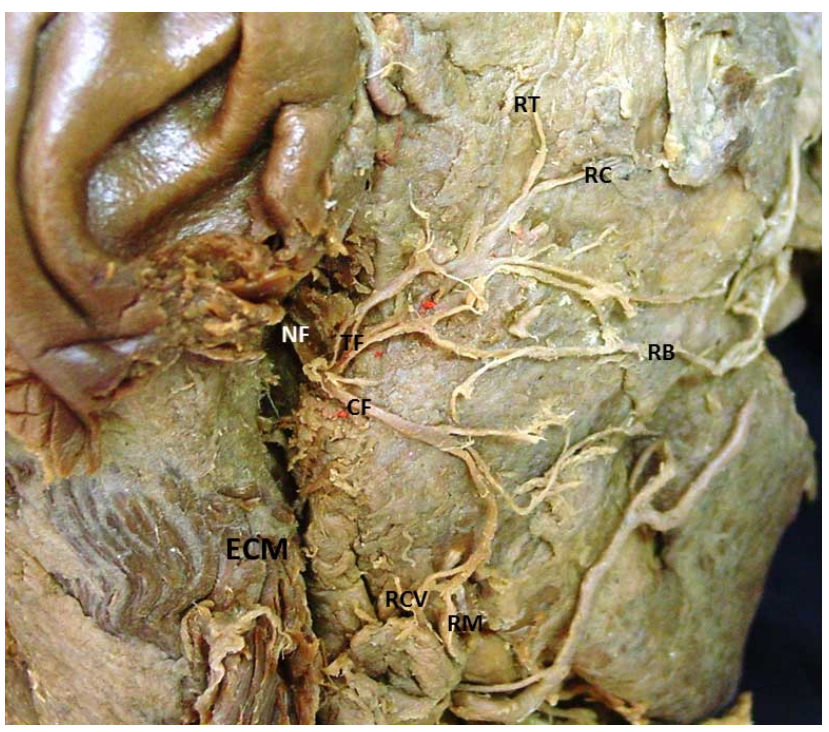

Fig. 4. Disección facial, se extrajo lobo superficial de GP para para observar nervio facial (NF) junto a sus 2 principales divisiones y ramos perifericos: temporofacial (TF) y cervicofacial (CF). Ramo frontotemporal (RT), ramo cigomático (RC), ramo bucal (RB), ramo marginal mandibular (RM) y ramo cervical (RCV). Musculo esternocleidomastoideo (ECM). 
El NF abandona la base de cráneo a través del foramen estilomastoideo (FEM) a $21+/-3.1 \mathrm{~mm}$ de la superficie cutánea (Kwak et al., 2004) (Fig. 3). Dos ramos consistentemente se originan desde el tronco del NF: el nervio auricular posterior destinado a inervar el vientre posterior del músculo occipitofrontal y al músculo auricular posterior, y el nervio del músculo digástrico para el músculo homónimo (Salame et al.). Ramos menos consistentes que se originan del tronco del NF al emerger por el FEM son: el ramo comunicante con el nervio glosofaríngeo y el nervio del músculo estilohioideo (Salame et al.). Luego el tronco del NF se dividirá en un ramo superior temporofacial (TF) y uno inferior cervicofacial (CF) (Fig. 4), esta bifurcación del NF en un estudio de disección de 46 lados de cara se produjo antes de la penetración en la GP en el 15\% de los casos y dentro de la glándula en el 85\% (Salame et al.). El NF separará anatómicamente a la GP en un lobo superficial y profundo (Fig. 2). La división TF originará a los ramos frontotemporales, cigomáticos y bucales. En cambio, la división $\mathrm{CF}$ dará origen al ramo marginal mandibular y cervical (Figs. 2 y 4 ).

Los ramos frontotemporales cruzan el hueso cigomático para inervar al vientre frontal del músculo occipitofrontal, músculo orbicular del ojo, músculo corrugador de la ceja y el músculo auricular superior y anterior. Los ramos cigomáticos estarán encargados de inervar el músculo cigomático mayor, cigomático menor, músculo elevador del labio superior y ala de la nariz, músculo elevador del labio superior y el músculo elevador del ángulo oral. Los ramos bucales transcurren paralelos al conducto parotídeo cruzando el músculo masetero para inervar al músculo buccinador, depresor del septo nasal y la mitad superior del músculo orbicular oral. Es frecuente encontrar comunicaciones entre el ramo TF y CF para formar una red de ramos bucales (Holsinger \& Bui, 2007) (Figs. 2 y 4).

El ramo marginal mandibular (RMM) se dirige por anterior e inferior de la GP para inervar al músculo depresor del ángulo oral, depresor del labio inferior y mentoniano (Figs. 2 y 4). Este RMM es una de las estructuras anatómicas más importantes cuando se realizan abordajes retromandibulares y/o submandibulares. Estudios anatómicos han demostrado que el nervio pasa por inferior del margen basilar de la mandíbula a una distancia de hasta $1,5 \mathrm{~cm}$ (Ziarah \& Atkinson, 1981).

La preparación del campo quirúrgico debe considerar la visualización de todo el lado facial intervenido para la evaluar la funcionalidad del NF, además se debe taponar con una gaza el meato acústico externo para evitar el ingreso de fluidos.
Dentro de los accesos descritos para la GP, la incisión Blair modificada es la más utilizada (Fig. 7). Una incisión alternativa es la de facelift modificada (Terris et al., 1994). Si una disección del cuello está prevista, la incisión de Blair modificada se puede adaptar fácilmente. Además, si el tumor se encuentra lateralmente al tronco principal del $\mathrm{NF}$, o si una exposición de su segmento intramastoideo es necesaria, la incisión de Blair modificada también se puede extender por retroauricular. El diseño de la incisión comienza por preauricular a nivel de la raíz del hélix, pudiendo aprovechar un pliegue pretragal descendiendo $1 \mathrm{~cm}$ por debajo del lóbulo. Desde este punto toma una forma arciforme, de concavidad anterior, procurando que esta curvatura sea suave para evitar necrosis del colgajo y algo alejada de la línea de implantación de la oreja. Realizada la curvatura la incisión ha de descender por posterior del margen anterior del músculo ECM, como a $1 \mathrm{~cm}$ de éste margen. La incisión desciende hasta alcanzar el extremo del asta mayor del hioides, pudiendo a veces hacerla coincidir con pliegues cutáneos. En su descenso se ha de conservar la distancia de al menos $1,5 \mathrm{~cm}$ con el margen basilar de la mandíbula para evitar lesión del RMM.

La incisión de piel se realiza con la hoja de bisturí a través del tejido subcutáneo y el músculo platisma. Cuidado se debe tener para no seccionar el nervio auricular mayor (NAM). El NAM es un ramo sensitivo del plexo cervical (C2 y C3) e inerva la porción inferior del oído externo y lóbulo de la oreja. El nervio viaja por posterior de la vena yugular externa, ambos por superficial al músculo ECM hasta el margen inferior de la GP, donde el NAM se divide en sus ramos anterior y posterior. Esta proximidad con la GP lo hace de gran utilidad como donante para injerto de NF, en casos de neoplasias malignas que infiltren el NF (Cueva et al., 1996).

Se prepara un colgajo anterior superficial al NAM y a la fascia parotídea. La fascia parotídea también llamada fascia parotidomaseterina debido a que también recubre al músculo masetero, le forma a la GP una cápsula avascular resistente (Holsinger \& Bui). La fascia parotídea es una prolongación superior de la lámina superficial de la fascia cervical profunda que recubre al músculo ECM y que al llegar a la GP se divide en una lamina superficial y profunda para recubrir completamente a la glándula. La lámina superficial que recubre a la GP es más gruesa y se continúa por superior con el arco cigomático.

Por lo que la elevación de un colgajo grueso se prefiere para disminuir la posibilidad del síndrome de Frey (Singleton \& Cassisi, 1980). Esto se logra siguiendo un plano avascular profundo a la lámina superficial de la fascia parotídea, de esta manera quedan protegidas las glándulas 
sudoríparas cutáneas de las fibras parasimpáticas seccionadas que son estimuladas cuando nos alimentamos. Acorde el colgajo es levantado por anterior de la GP los ramos periféricos del NF deben ser cuidadosamente evitados. Luego un colgajo posterior e inferior es elevado para exponer la cola de la GP. Después de elevados los colgajos estos pueden ser retraídos con suturas o ganchos de piel. La cola de la GP es disecada desde el músculo ECM a la profundidad del ramo posterior del NAM. La preservación de este último nervio depende del tipo de tumor, localización y extensión. Luego, el vientre posterior del músculo digástrico (VPMD) es expuesto con mayor elevación de la cola de la GP. Durante la elevación de la cola de la parótida, la integridad de la vena retromandibular (VRM) debe ser preservada si es posible. La VRM se forma dentro de la GP por la confluencia de las venas maxilares y la vena temporal superficial. La VRM atraviesa la GP profundo al NF y puede entregar una comunicante posterior intraparotídea que en confluencia con las venas occipitomastoideas formaran la vena yugular externa. La VRM extraparotídea se unirá con la vena facial para formar la vena facial común y así drenar en la vena yugular interna. La irrigación arterial de la GP proviene de ramos parotídeos de las arterias carótida externa, temporal superficial y maxilar, estructuras vasculares que también atravesaran la GP, profundas a la VRM. Por lo que estos elementos vasculares deben ser tomados en cuenta para el adecuado control de la hemorragia.

El espacio preauricular se abre dividiendo la unión de la GP de la porción cartilaginosa del meato acústico externo y así exponiendo el cartílago del trago. Este plano de disección creará un amplio campo desde el hueso cigomático hasta el VPMD y de esta manera la GP puede ser cuidadosamente retraída hacia anterior para identificar al NF.

El enfoque principal para identificar el NF depende del sitio y la extensión de la lesión. La preparación de todo el plexo, es decir, el tronco nervioso y de todos los ramos periféricos, se puede realizar bajo magnificación y estimulación eléctrica para reducir al mínimo el daño nervioso. Se describen tres posibles vías de abordaje:

I. Vía anterógrada: identificación del tronco del NF a su salida en el AEM. De esta manera la bifurcación y los diferentes ramos periféricos se identifican en una dirección de proximal a distal. Existen puntos de referencia anatómicos para identificar el tronco principal:

a) La fisura timpanomastoidea (FTM) a sido descrita como un punto de referencia anatómico fiable. Bushley et al. (2011) disecó 60 lados de cara en cadáveres, donde el tronco del NF se encontró en rangos de 3,3 a 9,2 mm con un promedio de 4,9 mm inferior a la FTM. Otro estudio realizado en cadáveres y en pacientes vivos, la distancia promedio de la FTM al tronco del NF fue de 1,8 (rango 0-4 mm) mm y 2 (rango 0-4) $\mathrm{mm}$ respectivamente (Witt et al., 2005).

b) El puntero tragal (PT) o de Conley es una extensión del cartílago del trago en el extremo medial de su margen anteroinferior. El tronco del NF en estudios anatómicos se encuentra $6.9+/-1.8 \mathrm{~mm}$ inferior y medial de este puntero (Rea et al., 2010) (Fig. 3).

c) El vientre posterior del músculo digástrico (VPMD) se encuentra en el mismo plano que el tronco del NF a 5,5 +/$2,1 \mathrm{~mm}$ por superior al músculo (Rea et al.). En cambio otro estudio en 22 pacientes vivos midieron la distancia desde el margen superior y posterior del VPMD al tronco del NF, la cual fue de 10,7 (rango 5-14) mm (Witt et al.) (Fig. 3).

d) El meato acústico externo cartilaginoso se encontraría 10,9+/ - 1,7 mm por sobre al tronco del NF (Witt et al.) (Fig. 3).

II. Vía retrógrada: De esta manera se identifican los ramos periféricos y la bifurcación del NF de distal a proximal. El mejor punto de referencia para comenzar con esta vía de abordaje es el tercio medio del arco cigomático donde la rama frontotemporal lo cruza (Gosain et al., 1997), este ramo se encuentra en una condensación de la fascia superficial, fascia temporal, y el periostio al cruzar el arco cigomático. Los ramos frontotemporales más posteriores se encuentran por delante del tubérculo articular del hueso temporal. Su ubicación se midió por Al-Kayat \& Bramley (1979) y fue de 3,5 +/- 0,8 cm desde el margen anterior del meato acústico externo óseo. Por otra parte el ramo cigomático se encuentra $1 \mathrm{~cm}$ por inferior del arco cigomático (Gosain, 1995). Alternativamente, la identificación del conducto parotídeo puede ser útil, ya que junto a él o por inferior transcurren en la mayoría de los casos los ramos bucales (Pogrel et al., 1996).

III. En muchos casos, una combinación de la vía anterógrada y retrógrada es necesaria. En cualquier caso, el cirujano tiene que ser entrenado en estas técnicas y conocer las variaciones anatómicas.

Una vez que el NF es identificado, la GP superficial al NF es dividida cuidadosamente, preservando la integridad del NF (Figs. 9 y 10). La localización exacta del NF debe ser previa a la separación del lobo superficial. Los tumores o masas pueden generar distorsión del NF y la GP.

El NF es preservado durante la parotidectomía excepto en casos de neoplasias malignas confirmadas, en las que el NF este infiltrado. En casos de invasión del NF por el cáncer, se realiza la resección. Los segmentos proximales y 
VELASCO, I.; SALINAS, F.; AGUILAR, L.; GALLEGO, A.; PASTRIÁN, J.; FARIÑA, R.; SOTO, R.; MEBUS, C. \& ZURBUCHEN, A. Consideraciones anatómicas en la parotidectomía: revisión de la literatura a propósito de un caso. Int. J. Morphol., 31(1):231-238, 2013.

distales deben ser examinados histológicamente por sección congelada para asegurar los márgenes quirúrgicos adecuados. Se puede realizar la reconstrucción nerviosa inmediata con un injerto de NAM o nervio sural. Finalmente los márgenes quirúrgicos son alineados y se sutura por planos, sobre un drenaje de mecanismo de acción cerrada si se prefiere.

\section{REPORTE DE CASO}

Paciente de 77 años, sexo masculino, consulta en el Servicio de Cirugía del Hospital del Salvador (Santiago, Chile) por un aumento de volumen en región parotídea derecha con 1 año de evolución, asintomático. Como antecedentes médicos el paciente padecía de hipertensión arterial controlada y era fumador de tabaco crónico. Al examen clínico se observó tumoración parotídea derecha de unos $8 \mathrm{~cm}$ aproximadamente de diámetro que causaba evidente asimetría facial, la piel que compromete la lesión no mostraba alteraciones (Fig. 5). A la palpación la masa era de consistencia firme, bien delimitada, no dolorosa y sin adherencia a la piel. No se palparon adenopatías cervicales o locales. La función del nervio facial se encontraba intacta.

Se solicitó como examen imagenológico inicial ecografía parotídea, que mostró lesión hipogénica, nodulada en lobo superficial de la GP (Fig. 6a). También se realizó tomografía computada (TC) maxilofacial para observar la

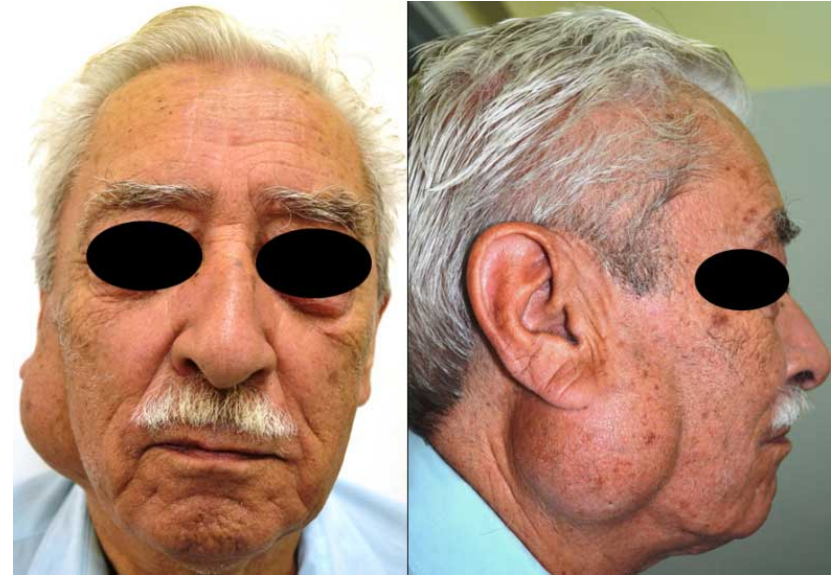

Fig. 5. Visión anterior y lateral de tumoración parotídea.

extensión de la lesión (Fig. 6b). En base a los hallazgos clínicos e imagenológicos nuestra hipótesis diagnóstica es de una neoplasia benigna concordante con el adenoma pleomorfo.

Se planificó parotidectomía superficial bajo anestesia general. Mediante incisión de Blair modificada (Fig. 7), se identificó por vía anterógrada al NF usando como referencia anatómica al VPMD y al puntero tragal (Fig. 8). Luego se realizó eliminación total del tumor cuidando la integridad de todos los ramos del NF (Fig. 9). Finalmente se cerró por planos con instalación de drenaje aspirativo.

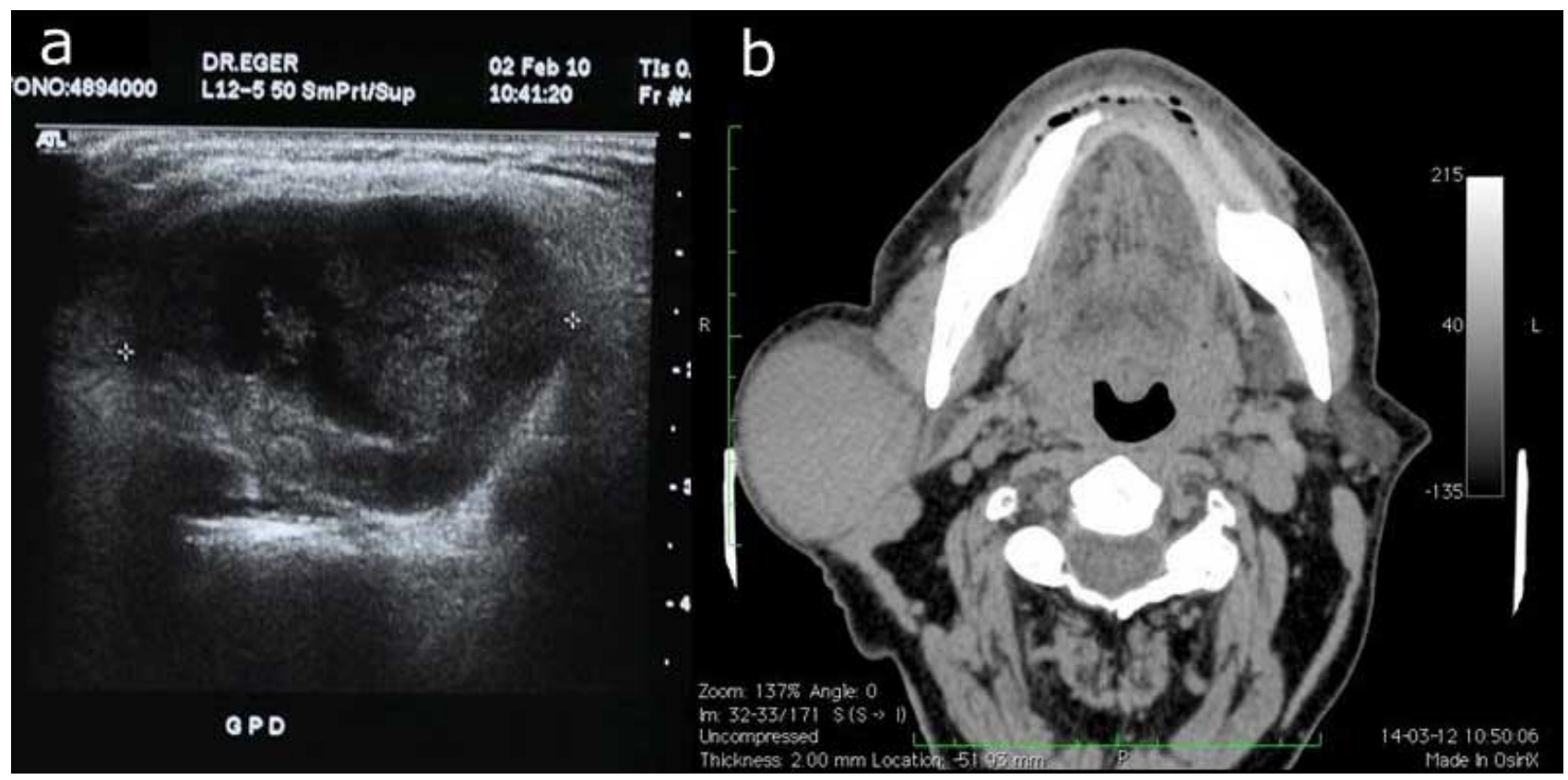

Fig. 6a. Ecografía parotídea que muestra lesión hipogénica y nodular en lobo superficial. 6b. TC sin contraste con ventana de tejidos blandos, corte axial muestra extensa tumoración en GP derecha que media $5 \times 5 \mathrm{~cm}$. 


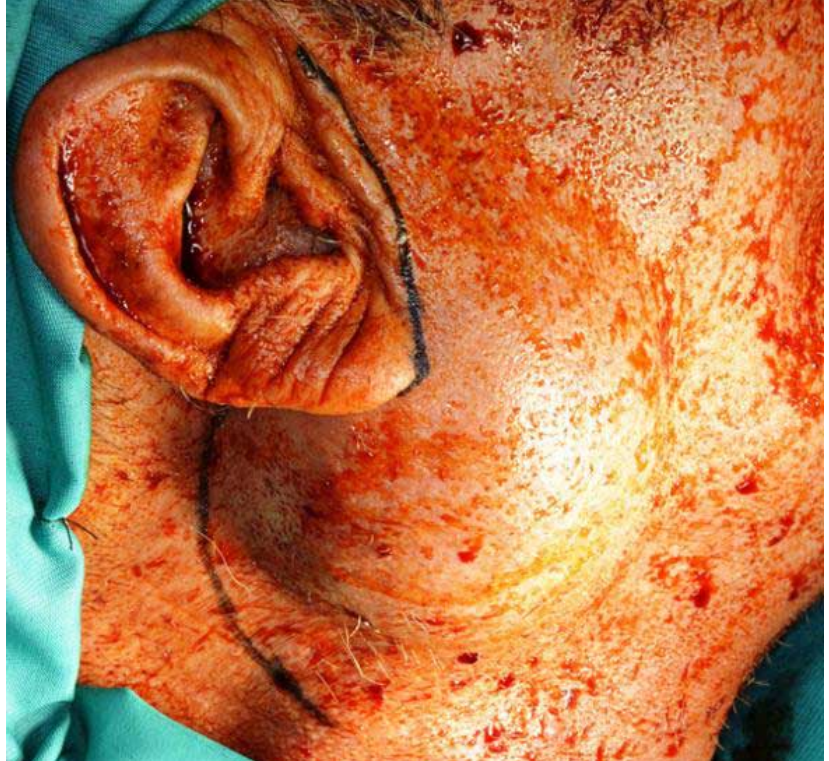

Fig. 7.Incisión de Blair modificada.

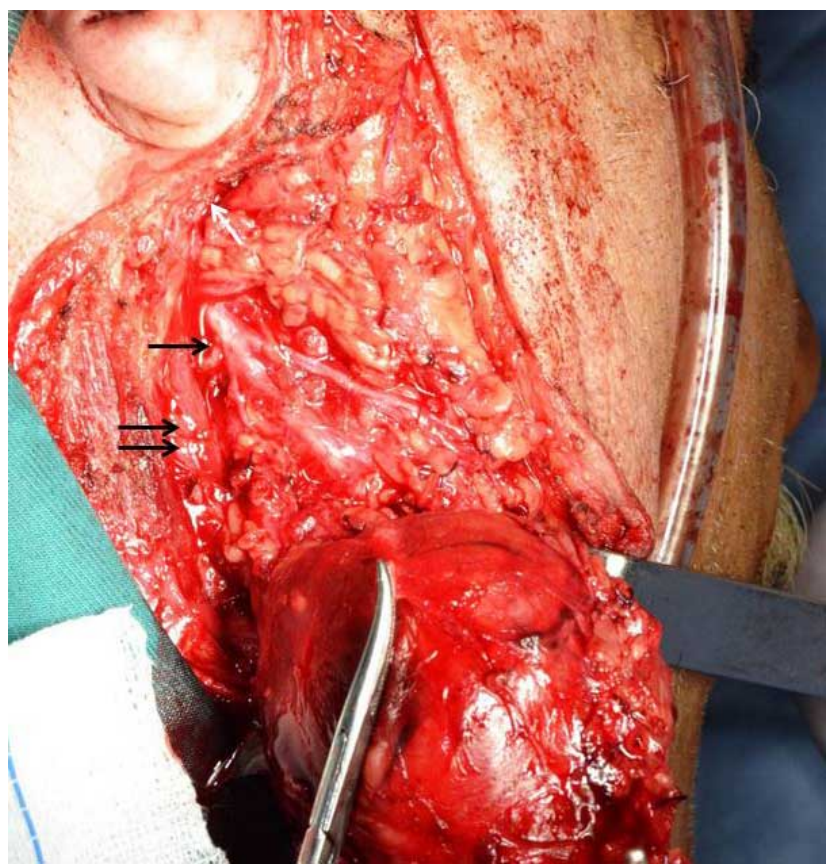

Fig. 8.Identificación del NF (flecha negra) tomando como referencias anatómicas al VPMD (doble flecha) y al puntero tragal (flecha blanca) previo a parotidectomía superficial y exéresis total del tumor.

La biopsia del espécimen quirúrgico entregó el diagnóstico final de cistoadenoma papilar linfomatoso (tumor de Warthin) (Fig. 10). Seguimientos post-operatorios no mostraron signos de infección y la función del NF se encontraba intacta. El paciente seguirá bajo controles periódicos para identificar alguna recurrencia.

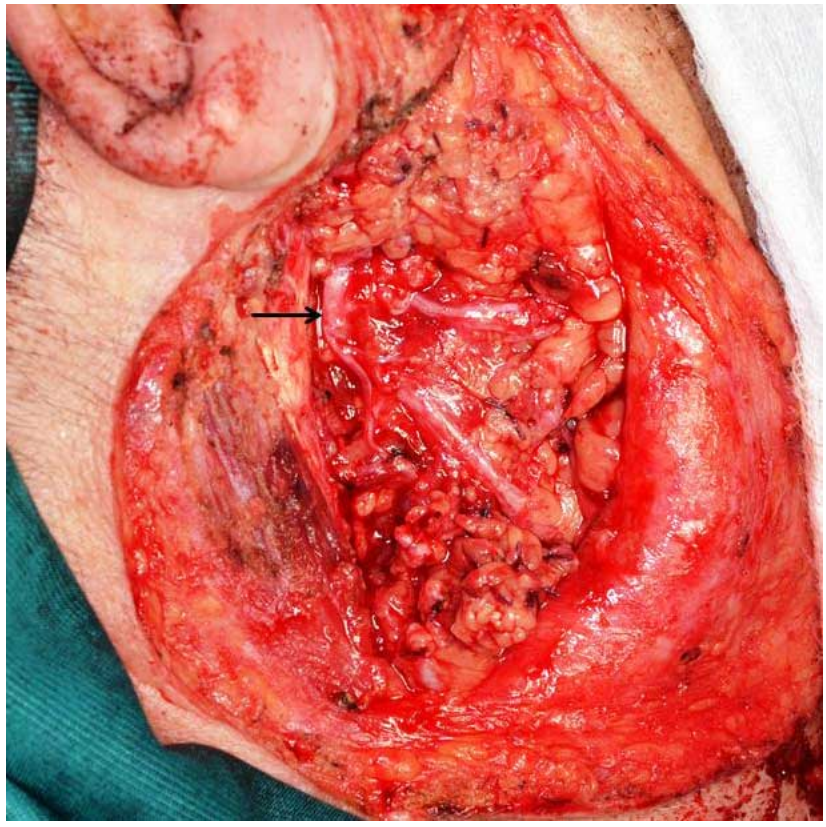

Fig. 9.Parotidectomía superficial preservando integridad del NF y de sus ramos (flecha).

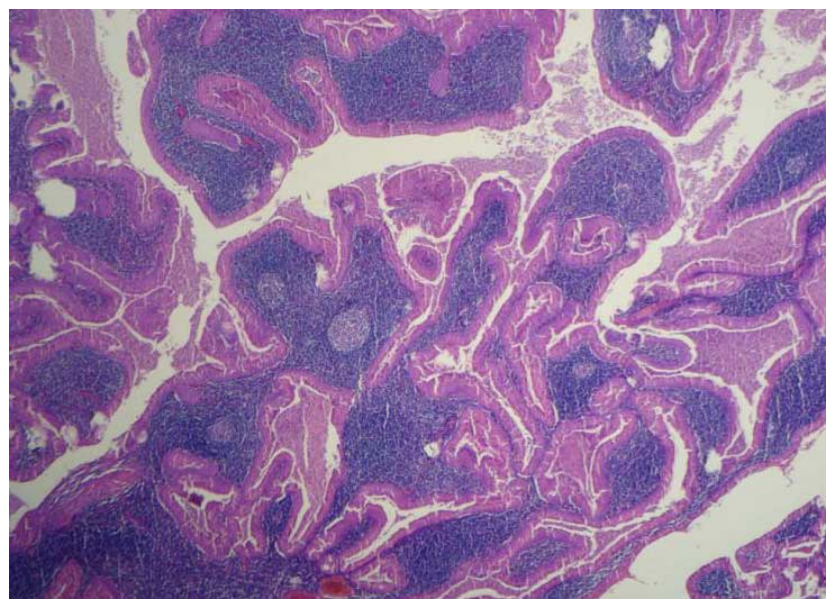

Fig. 10. Histopatología muestra el componente epitelial oncocítico junto al característico estroma linfoide con centros germinales. Tinción hematoxilina-eosina, magnificación original 60X.

\section{DISCUSIÓN}

Las neoplasias de GP constituyen el 3\% de todas las neoplasias de cabeza y cuello (Horn-Ross et al., 1991), siendo la gran mayoría de naturaleza benigna (Spiro, 1986). El tumor mas común es el adenoma pleomorfo el cual representa el 80\% de todas las neoplasias benignas de GP (Spiro).

El tumor de Warthin es la segunda neoplasia benigna mas prevalente en GP contando el 6-10\% del total de los 
tumores (Ellis \& Auclair, 1996). Se encuentra casi exclusivamente en la GP y la mayoría de los casos involucran el polo inferior de la glándula. Clínicamente se presenta como una masa de crecimiento lento y progresivo de 2 a $4 \mathrm{~cm}$ de tamaño; tumores de mayor tamaño son excepcionales (Ellis \& Auclair). La etiología de esta neoplasia no es clara pero se la ha dado una fuerte asociación a los pacientes fumadores, que tendrían una incidencia 8 veces mayor que los no fumadores (Kotwall, 1992).

La resección quirúrgica es el tratamiento de elección para las neoplasias parotídeas, pero éstas varían desde la enucleación del tumor a la parotidectomía total, dependiendo de su extensión y naturaleza (Witt, 2002). El lobo superficial de la GP, ubicado lateral al tronco del NF y de sus ramos, contiene el $80 \%$ del parénquima glandular y la mayoría de los linfonodos parotídeos (Holsinger \& Bui). Consecuentemente, la gran mayoría de tumores parotídeos están ubicados en el lobo superficial. Por lo tanto, la parotidectomía superficial es el tratamiento quirúrgico de elección para la mayoría de neoplasias benignas (Laskawi et al., 1996).

La real utilidad de procedimientos quirúrgicos menos extensos es un asunto de continua controversia. Los resultados de recientes meta-análisis publicados muestran que sólo la enucleación de tumores parotídeos es 9 veces más probable de producir recurrencia que la parotidectomía superficial (Witt et al.). En el caso del tumor de Warthin el tratamiento a través de la parotidectomía parcial-superficial o la resección local es igualmente curativo (Iwai \& Yamashita, 2005). El tumor de Warthin posee una baja tasa de recurrencia, y en la mayoría de los reportes va en rangos de $2 \%$ a $5,5 \%$ (Ellis \& Auclair). Los cambios malignos son muy infrecuentes y son vistos en menos del $1 \%$ de los casos, pudiendo comprometer tanto el componente epitelial como el linfoide (Warnock, 1991).

Sin embargo, la parotidectomía no es un procedimiento benigno. Los riesgos incluyen hemorragia, infección de la herida, fístula salival, sialocele, necrosis del colgajo, síndrome de Frey, parálisis temporal o permanente del NF, secuela cosmética, déficit sensitivo del pabellón auricular, síndrome de primera-mordida y recurrencia de la neoplasia. (Wang \& Eisele, 2012; Harell et al., 1996; Thompson \& McCffrey, 1996). Chedid et al., (2011) publicaron una revisión de 70 casos de resecciones quirúrgicas de tumores de Warthin donde un 54\% de los pacientes no presentó complicación alguna. Aunque éstas son poco frecuentes, la parálisis del NF es incuestionablemente la más devastadora y la más frecuente. La literatura actual cita que la tasa de parálisis del NF después de la parotidectomía va en rangos de 0$7 \%$ y la paresia temporal hasta $64 \%$ (Lowry et al., 2005).
La incidencia de seromas generalmente es baja, sin embargo Chedid et al., (2011) reportaron que el 13,7\% de sus pacientes sometidos a parotidectomía presentaron esta complicación, ocupando el segundo lugar después de la parálisis del NF. Esto puede deberse al tipo de drenaje utilizado ya sea un Penrose o un drenaje de aspiración cerrada, siendo este último el más aceptado actualmente. Se cree que la causa podría radicar en el retiro anticipado del drenaje y la ausencia de apósitos compresivos sobre la zona intervenida (Chedid et al.).

Se reporta que la mayoría de los pacientes tratados por parotidectomía podrían presentar algún signo de síndrome de Frey. De hecho esta corresponde a la tercera complicación más frecuente (Chedid et al.). Sin embargo, solo el $10 \%$ se queja de molestias asociadas (Wang \& Eisele). Se cree, que la etiología está radicada en la regeneración aberrante de fibras nerviosas parasimpáticas postganglionares secretomotoras (del nervio auriculotemporal) que inervan a la GP y que, posterior al procedimiento, se unirián a fibras simpáticas postganglionares dañadas que inervan a glándulas sudoríparas dérmicas de la cara. Así, cuando el individuo se alimenta y estimula la salivación se desencadena la hiperhidrosis e hiperemia local.

En el caso reportado en esta publicación no se ha presentado ninguna complicación hasta la fecha, sin embargo es necesario continuar los controles posteriores para identificar cualquier complicación tardía que pueda presentarse.

VELASCO, I.; SALINAS, F.; AGUILAR, L.; GALLEGO, A.; PASTRIÁN, J.; FARIÑA, R.; SOTO, R.; MEBUS, C. \& ZURBUCHEN, A. Anatomical considerations in parotidectomy: review of the literature and case report. Int. J. Morphol., 31(1):231$238,2013$.

SUMMARY: Parotidectomy is a surgical procedure usually performed by Oral and Maxillofacial Surgeons and Head and Neck Surgeons, in which the parotid gland may be removed partially or totally. The most common indications for this procedure are the neoplasms of the gland. Approximately $80 \%$ of total salivary gland tumors occur in the parotid gland. Of these, $75-80 \%$ are benign. However, the parotidectomy is a highly complex procedure because of the regional anatomy and the close relationship of the parotid gland with the facial nerve. The most important aspect of this procedure is the functional preservation of the facial nerve and its branches. Therefore, the thorough knowledge of the anatomy of the parotid gland and related structures is necessary for surgical success. This review and case report will discuss the anatomical considerations that must be taken during parotidectomy to reduce the possibility of complications.

KEY WORDS: Parotid gland; Parotid region; Parotidectomy, Facial nerve; Warthin's tumor. 


\section{REFERENCIAS BIBLIOGRÁFICAS}

Al-Kayat, A. \& Bramley, P. A. A modified pre-auricular approach to the temporomandibular joint and malar arch. Br. J. Oral Surg., 17:91$103,1979$.

Bushey, A.; Quereshy, F.; Boice, J. G.; Landers, M. A. \& Baur, D. A. Utilization of the tympanomastoid fissure for intraoperative identification of the facial nerve: a cadaver study. J. Oral Maxillofac. Surg., 69(9):2473-6, 2011.

Cueva, R. A.; Robbins, K. T. \& Martin, P. J. Lower cervical cutaneus sensory nerves: An alternative for facial nerve cable grafting. Otolaryngol. Head Neck Surg., 114:479-81, 1996.

Chedid, H. M.; Rapoport, A.; Aikawa, K. F.; Menezes, A. S. \& Curioni, A. O. Warthin tumor of the parotid gland: a study of 70 cases. Rev. Col. Bras. Cir., 38(2):90-4, 2011.

Ellis, G. L., Auclair, P. L. Warthin's tumor (papillary cystadenoma lymphomatosum). In: Atlas of Tumor Pathology: Tumors of the Salivary Glands. Washington, D. C. Armed Forces Institute of Pathology, 1996. pp.68-79. 3rd series, fascicle 17.

Futran, N. D.; Parvathaneni, U.; Martins, R. G. \& Laramore, G. E. Malignant salivary gland tumors. In: Head and neck cancer: a multidisciplinary approach. Harrison, L. B.; Sessions, R. B. \& Hong, W. K. (eds). Philadelphia, Lippincott Williams \& Wilkins, 2009. pp. 589-610.

Gosain, A. K. Surgical anatomy of the facial nerve. Clin. Plast. Surg., 22:241-51, 1995

Gosain, A.K.; Sewall, S. R. \& Yousif, N. J. The temporal branch of the facial nerve: how reliably can we predict its path? Plast. Reconstr. Surg., 99:1224-36, 1997.

Harell, M.; Levy, D. \& Elam, M. Superficial parotidectomy for benign parotid lesions. Oper. Tech. Otolaryngol. - Head Neck Surg., 7(4):31522, 1996.

Holsinger, F. C. \& Bui, D. T. Anatomy, function, and evaluation of salivary glands. In: Salivary Gland Disorders. Myers, E. N., Ferris, R. L. (eds.). 1st. Ed. Heidelberg, Germany: Springer-Verlag, 2007. pp. 1-16.

Horn-Ross, P. L.; West, D. W. \& Brown, S. R. Recent trends in the incidence of salivary gland cancer. Int. J. Epidemiol., 20:628, 1991.

Iwai, H. \& Yamashita, T. Local excision procedure for Warthin; s tumor of the parotid gland. Otolaryngol. Head Neck Surg., 132(4):577-80, 2005.

Kotwall, C. A. Smoking as an etiologic factor in the development of Warthin's tumor of the parotid gland. Am. J. Surg., 164(6):646-7, 1992.

Kwak, H. H.; Park, H. D.; Youn, K. H.; Hu, K. S.; Koh, K. S.; Han, S. H. \& Kim, H. J. Branching patterns of the facial nerve and its communication with the auriculotemporal nerve. Surg. Radiol. Anat., 26:494-500, 2004.

Laskawi, R.; Schott, T. \& Mirzaie-Petri, M. Surgical Management of pleomorphic adenomas of the parotid gland: A follow up study of three methods. J. Oral Maxillofac. Surg., 54:1176, 1996.
Leverstein, H.; van der Wal, J. E.; Tiwari, R. M.; van der Waal, I. \& Snow, G. B. Surgical management of 246 previously untreated pleomorphic adenomas of the parotid gland. Br. J. Surg., 84:399-403, 1997.

Lowry, T. R., Gal, T. J. \& Brennan, J. A. Patterns of use of facial nerve monitoring during parotid gland surgery. Otolaryngol. Head Neck Surg.,133(3):313-8, 2005.

Pather, N. \& Osman, M. Landmarks of the facial nerve: implications for parotidectomy. Surg. Radiol. Anat., 28(2):170-5, 2006.

Pogrel, M. A.; Schmidt, B. \& Ammar, A. The relationship of the buccal branch of the facial nerve to the parotid duct. J. Oral Maxillofac. Surg., 54(1):71-3, 1996.

Rea, P. M.; McGarry, G. \& Shaw-Dunn, J. The precision of four commonly used surgical landmarks for locating the facial nerve in anterograde parotidectomy in humans. Ann. Anat., 192:27-32, 2010.

Salame, K.; Ouaknine, G.; Arensburg, B. \& Rochkind, S. Microsurgical anatomy of the facial nerve trunk. Clin. Anat., 15:93-9, 2002.

Singleton, G. T. \& Cassisi, N. J. Frey's syndrome: Incidence related to skin flap thickness in parotidectomy. Laryngoscope, 90:1636-9, 1980.

Spiro, R. H. Salivary neoplasms: Overview of 35-year experience with 2,807 patients. Head Neck Surg., 8:177, 1986.

Terris, D. J.; Tuffo, K. M. \& Fee, W. E. Jr. Modified facelift incision for parotidectomy. J. Laryngol Otol., 108:574-8,1994.

Thompson, D. \& McCaffrey ,T. Surgical approach for primary parotid malignancies. Oper. Tech. Otolaryngol. - Head Neck Surg., 7(4):35864, 1996.

Wang, S. J. \& Eisele, D. W. Parotidectomy - Anatomical considerations. Clin. Anat, 25(1):12-8, 2012

Warnock, G. R. Papillary cystadenoma lymphomatosum (Warthin's tumor). In: Surgical Pathology of the Salivary Glands. Ellis, G. L., Auclair, P. L. \& Gnepp, D. R. (eds.). Philadelphia, WB Saunders, 1991. pp.187201.

Witt, R. L. The significance of the margin in parotid surgery for pleomorphic adenoma. Laryngoscope, 112:2141, 2002.

Witt, R. L.; Weinstein, G. S. \& Rejto, L. K. Tympanomastoid suture and digastric muscle in cadaver and live parotidectomy. Laryngoscope, 115:574-7, 2005

Ziarah, H. Á. \& Atkinson, M. E. The surgical anatomy of the cervical distribution of the facial nerve. Br. J. Oral Surg., 19:171-9, 1989.

\section{Dirección para correspondencia:}

Dr. Ignacio Velasco Martínez

Servicio de Cirugía Maxilofacial

Hospital del Salvador.

Dirección: Av. Salvador 364, Providencia

Santiago - CHILE

E-mail: iavelasco@miuandes.cl

Recibido : 19-04-2012

Aceptado: 29-08-2012 